Working Paper 10-16

Business Economic Series 04

April 2010
Departamento de Economía de la Empresa Universidad Carlos III de Madrid

Calle Madrid, 126

28903 Getafe (Spain)

Fax (34-91) 6249607

\title{
Derivatives Pricing with Marked Point Processes Using Tick-by-Tick Data *
}

\author{
Alvaro Cartea $^{1}$
}

\begin{abstract}
I propose to model stock price tick-by-tick data via a non-explosive marked point process. The arrival of trades is driven by a counting process in which the waiting-time between trades possesses a Mittag-Leffler survival function and price revisions have an infinitely divisible distribution. I show that the partial-integro-differential equation satisfied by the value of European-style derivatives contains a non-local operator in time-to-maturity known as the Caputo fractional derivative. Numerical examples are provided for a marked point process with conditionally Gaussian and with conditionally CGMY price innovations. Furthermore, the infinitesimal generator of the marked point process derived to price derivatives coincides with that of a Lévy process of either finite or infinite activity.
\end{abstract}

Keywords: Tick-by-tick data, waiting-times, duration, high frequency data, Caputo operator, marked point process.

JEL Classification: G12, G13, C41 


\title{
Derivatives Pricing with Marked Point Processes using Tick-by-Tick Data
}

\author{
Álvaro Cartea*
}

April 6, 2010

\begin{abstract}
I propose to model stock price tick-by-tick data via a non-explosive marked point process. The arrival of trades is driven by a counting process in which the waiting-time between trades possesses a Mittag-Leffler survival function and price revisions have an infinitely divisible distribution. I show that the partial-integro-differential equation satisfied by the value of European-style derivatives contains a non-local operator in time-to-maturity known as the Caputo fractional derivative. Numerical examples are provided for a marked point process with conditionally Gaussian and with conditionally CGMY price innovations. Furthermore, the infinitesimal generator of the marked point process derived to price derivatives coincides with that of a Lévy process of either finite or infinite activity.
\end{abstract}

Keywords: Tick-by-tick data, waiting-times, duration, high frequency data, Caputo operator, marked point process.

JEL Classifications: G12, G13, C41

\footnotetext{
*Universidad Carlos III de Madrid. alvaro.cartea@uc3m.es. This paper has benefited from comments of seminar participants at The University of Chicago, University of Florence, University of Toronto, Fields Institute University of Oxford, ESSEC, King's College London, Birkbeck-University of London and Universidad Carlos III de Madrid. For comments and suggestions on earlier drafts, I am indebted to G. Amromin, R. Brummelhuis, H. Geman, S. Howison and L.P. Hughston. In particular, I would like to give special thanks to S. Jaimungal for very useful and insightful comments at different stages of this paper.
} 


\section{Introduction}

Access to better and more financial data is a constant source of new research and allows us to refine solutions to old problems and to extract market information that previously could not be obtained. A striking example is the relatively recent access to high-frequency data. Nowadays it is possible to find equity data with information on every trade including: time-stamp to nearest second, volume, quotes, etc. One of the many applications is, for instance, to further the understanding of stock price dynamics during 'business time' instead of 'calendar time'; an insight first proposed in Clark (1973).

In a recent paper, Cartea and Meyer-Brandis (2010) employ tick-by-tick data to propose a model that explicitly uses information on the waiting-time between trades. In their model the arrival of trades is determined by a counting process that captures the empirical waiting-time distribution under the data generating measure. In this paper I further investigate the pricing equations arising from a model that explicitly incorporates information on the waiting-time between trades. I assume that the $\log$ stock dynamics are represented by a marked point process where the counting process $N(t)$ represents the number of trades and the marks $Y$ are i.i.d. random variables that represent price revisions. The price revisions $Y$ possess an infinitely divisible distribution with log-characteristic function given by the Lévy-Khintchine formula:

$$
\ln \mathbb{E}\left[e^{i \xi Y}\right] \equiv \Psi(\xi)=a i \xi-\frac{1}{2} \sigma^{2} \xi^{2}+\int_{\mathbb{R}_{0}}\left(e^{i \xi y}-1-i \xi u(y)\right) W(d y),
$$

where $a \in \mathbb{R}, \sigma \geq 0, u(y)$ is a truncation function and $\Psi(\xi)$ is the characteristic exponent of the Lévy distribution with triplet $\left(a, \sigma^{2}, W\right)$.

Modeling price dynamics with a marked point process is related to the vast literature on time changes and stochastic volatility (see for example Heston (1993), Kallsen and Shiryaev (2002), Carr, Geman, Madan, and Yor (2003), Carr and Wu (2004) and Cartea and Howison (2009)) in the sense that the market undergoes periods of high activity and low activity. In the model used here the 'pace' of the market is driven by the arrival of trades, whereas in stochastic volatility models, which can be seen as time-changed Brownian motions, periods of high (resp. low) volatility are periods of high (resp. low) activity in the market. In other words, marked point processes and stochastic volatility models can both 
be viewed as models that reflect the market's trading activity which is better measured in business time rather than calendar time; an effect also captured in time-changed Brownian motion models.

I highlight two new and significant contributions of this paper. First, I show that the value of European-style options satisfy a partial integro-differential equation (PIDE) in time-to-maturity where, instead of the traditional $\partial / \partial T$ derivative, the time-to-maturity derivative is a non-local operator known as the Caputo operator. The Caputo nonlocal operator is a fractional derivative which is an interpolation between integer-order derivatives that nests the usual local derivative $\partial / \partial T$ as a particular case. I derive the result by using an infinite order expansion of the characteristic function of the log-price dynamics. These pricing PIDEs are new in the literature and result from modeling price dynamics that incorporate data to capture the stochastic: i) arrival of trades, and ii) price revisions every time there is a trade. This approach profits from the knowledge provided by tick-by-tick equity data; information that the traditional continuous-time models do not exploit.

Second, I show that the infinitesimal operator appearing in the pricing PIDEs coincides with the infinitesimal operator of a Lévy process. At first this is expected because, after all, it is assumed that the price shocks possess an infinitely divisible distribution. But if we bear in mind that the family of waiting-time distributions used here can only have a finite number of trades within a finite time interval, it is interesting to see that we obtain pricing equations with an infinitesimal generator of a Lévy process that includes infinite activity processes.

The rest of the paper is organized as follows. Section 2 provides the necessary definitions to introduce a marked point process and presents the stock price dynamics used throughout the paper. Section 3 discusses a particular waiting-time distribution and survival function in the light of tick-by-tick data. Section 4 derives and discusses the main result of the paper. Section 5 presents some numerical examples of European call option prices under two different marked point processes. Finally, Section 6 concludes. 


\section{Price dynamics as a Marked Point Process}

A popular way to model stock price dynamics is to assume that under the risk-neutral measure prices are driven by a Lévy process. In particular, it is assumed that stock prices $S(t)$ satisfy the stochastic differential equation

$$
d \ln S(t)=(r-D-c) d t+d L_{t}^{Q}
$$

where $r$ is a constant risk-free rate, $D$ is the continuously compounded dividend yield, $c$ is a convexity adjustment so that $\mathbb{E}\left[S(T) \mid \mathcal{F}_{t}\right]=S(t) e^{(r-D)(T-t)}$, where $\mathbb{E}\left[\cdot \mid \mathcal{F}_{t}\right]$ is the expectation operator conditioned on information at time $t$, and $L_{t}^{Q}$ is a Lévy process with triplet $\left(0, \sigma_{o}^{2}, W_{o}\right)$ and log-characteristic function given by $t \Psi(\xi)$, see (1.1).

It is a well-known result that if stock prices follow (2.1) then the value of a European-style option, denoted by $V(x, t)$, with payoff $G(x(T))$ is given by $e^{-r(T-t)} \mathbb{E}\left[G(x(T)) \mid \mathcal{F}_{t}\right]$ which satisfies the PIDE, in time-to-maturity,

$$
\begin{aligned}
& -\frac{\partial V(x, T)}{\partial T}+\frac{1}{2} \sigma_{o}^{2} \frac{\partial^{2} V(x, T)}{\partial x^{2}}+(r-D-c) \frac{\partial V(x, T)}{\partial x} \\
+ & \int_{\mathbb{R}}\left(V(x+y, T)-V(x, T)-u(y) \frac{\partial V(x, T)}{\partial x}\right) W_{o}(d y)=r V(x, T),
\end{aligned}
$$

subject to an initial condition, see Cont and Tankov (2004).

Modeling spot price dynamics with (2.1) assumes that there is a trade at each instant in time. We know, however, that although there are stocks that trade very frequently, there are time intervals when no trades take place, and more importantly, we observe that the time between consecutive trades is stochastic. Access to tick-by-tick data, with a time-stamp for every trade, allows us to depart from the traditional approach that assumes continuous trading to one where we can incorporate information on waiting times between trades. Ideally, we want a model that can build on high-frequency data with information on times of trades as well as price revisions. And, indeed, the theory of marked point processes provides the right framework to model processes where there are events that occur at random times (trades) with a mark (price revision) associated to each event. 
Below I define the different elements required to model the log-stock risk-neutral dynamics of spot prices within the theory of point processes. I define a simple point process, a marked point process and then show that the discounted log-stock process used here is a martingale. For a comprehensive treatment of point processes see Jacobsen (2006).

First some notation. Let $(\Omega, \mathcal{F}, \mathbb{P})$ be a probability space with sample space $\Omega$ a non-empty set, $\mathcal{F}$ a $\sigma$-algebra of subsets of $\Omega$, and $\mathbb{P}$ a probability measure on $\mathcal{F}$ and let $\overline{\mathbb{R}}_{0}=[0, \infty]$. Moreover, suppose we are given a measurable space $(E, \mathcal{E})$ called the mark space. Adjoin to $E$ the irrelevant mark $\nabla$, to be used for describing the mark of an event that never occurs, write $\bar{E}=E \cup\{\nabla\}$ and let $\overline{\mathcal{E}}=\sigma(\mathcal{E},\{\nabla\})$ denote the $\sigma$-algebra of subsets of $\bar{E}$ generated by the measurable subsets of $E$ and the singleton $\{\nabla\}$.

Definition 2.1 Simple Point Process. A simple point process $(S P P)$ is a sequence $\mathcal{T}=\left(T_{n}\right)_{n \geq 1}$ of $\overline{\mathbb{R}}_{0}$-valued random variables defined on $(\Omega, \mathcal{F}, \mathbb{P})$ such that

1. $\mathbb{P}\left(0<T_{1} \leq T_{2} \leq \cdots\right)=1$,

2. $\mathbb{P}\left(T_{n}<T_{n+1}, T_{n}<\infty\right)=\mathbb{P}\left(T_{n}<\infty\right) \quad(n \geq 1)$,

3. $\mathbb{P}\left(\lim _{n \rightarrow \infty} T_{n}=\infty\right)=1$.

The definition includes condition 3 because for the particular waiting-time distribution employed in the model below, there can only be a finite number of trades in a finite time interval. (Condition 3 can be relaxed to include SPP with explosions, see Jacobsen (2006) for details.)

Definition 2.2 Marked Point Process. A marked point process (MPP) with mark space E is a double sequence $(\mathcal{T}, \mathcal{Y})=\left(\left(T_{n}\right)_{n \geq 1},\left(Y_{n}\right)_{n \geq 1}\right)$ of $\overline{\mathbb{R}}_{+}$-valued random variables $T_{n}$ and $\bar{E}$-valued random variables $Y_{n}$ defined on $(\Omega, \mathcal{F}, \mathbb{P})$ such that $\mathcal{T}=\left(T_{n}\right)$ is an SPP and

1. $\mathbb{P}\left(Y_{n} \in E, T_{n}<\infty\right)=\mathbb{P}\left(T_{n}<\infty\right) \quad(n \geq 1)$,

2. $\mathbb{P}\left(Y_{n}=\nabla, T_{n}=\infty\right)=\mathbb{P}\left(T_{n}=\infty\right) \quad(n \geq 1)$.

We are also interested in the counting process $N=\left(N_{t}\right)_{t \geq 0}$ associated to the $\operatorname{SPP} \mathcal{T}=\left(T_{n}\right)_{n \geq 1}$ which we define as $N(t)=\sum_{n=1}^{\infty} 1_{T_{n} \leq t}$. In the model $N(t)$ counts the number of trades over the time interval $[0, t]$ with $N(0)=0$. 


\section{The Model for tick-by-tick spot data}

The risk-neutral dynamics of log-stock prices are given by

$$
d X(t)=(r-D) d t+Y d N(t)
$$

where $X(t)=\ln S(t), r$ is the risk-free rate, $D$ is the dividend yield, $N(t)$ is a counting process and $Y$ are i.i.d. price revisions with infinitely divisible distribution and triplet $\left(w, \sigma^{2}, W\right)$, independent of $N(t)$, where $w$ is such that $\mathbb{E}\left[e^{Y}-1\right]=0$.

The novel part of this model for risk-neutral dynamics is the stochastic component in equation 2.3

$$
U(t)=\sum_{n=1}^{N(t)} Y_{i}
$$

which is an $\mathbb{R}$-valued MPP with jump times $T_{n}$, jump sizes $\left(Y_{n}\right)$ with distribution $g$ and $N(t)$ is a counting process independent of the i.i.d. $Y$. In the simple case where $N(t)$ is a homogeneous Poisson process with rate $\lambda>0$, the MPP $U(t)$ is a Lévy process with bounded Lévy measure $\lambda g$.

In model (2.3) we can distinguish two components: a deterministic one, which is the drift $(r-D) t$, and a stochastic one, which is the jump component $\sum_{i=1}^{N(t)} Y_{i}$. Moreover, since we are interested in financial applications where we use tick-by-tick data, we examine further some of the building blocks of the arrival of trades $N(t)$ : the waiting-time between trades, the survival function, and the rate of arrival of trades, i.e. the hazard rate.

Define the waiting times between trades by $T_{n}-T_{n-1}=\tau_{n}$ and assume that the waiting-time possesses a continuous pdf $v(t)$. Hence we can write

$$
T_{n}=T_{0}+\sum_{i=1}^{n} \tau_{i}, \quad T_{n}-T_{n-1}=\tau_{n}, \quad n=1,2,3, \cdots
$$

and the survival function, which represents the probability that the waiting-time between two consecutive trades is greater than $t$, is given by

$$
\Upsilon(t)=1-\int_{0}^{t} v(u) d u
$$


Finally, the hazard function $u(t)$ is defined as

$$
u(t)=-\frac{d}{d t} \ln \Upsilon(t), \quad t \in \overline{\mathbb{R}}_{0}
$$

Intuitively, the hazard function represents the probability that a trade will happen in the next small time interval divided by the length of that time interval; i.e. the hazard function is the instantaneous intensity of a trade occurrence. Here I assume that $u(t)$ is strictly positive and continuous.

In the model, only when we assume that the distribution of the waiting times is exponential do we find that stock prices are Markovian; the MPP $U(t)$ becomes a compound Poisson process. In all other cases the log-stock price process $X(t)$ in (2.3) is not Markovian for a general waiting-time distribution because the probability of observing a trade over the next time-step depends on how long we have been waiting since the last trade. Denote by $H(\omega, t)=H(t)$ the so-called backward recurrence time (i.e. the time elapsed since the last trade) which is defined by

$$
H(t):=t-T_{N_{t}}
$$

and where $T_{N_{t}}$ represents the last trade time before $t$. Then the intensity of the counting process $N(t)$ is given by $u(H(t))$ and the predictable compensator of the jump measure $N(d t, d z)$ is the random measure

$$
v(\omega, d t, d z)=v(d t, d z):=u(H(t)) g(z) d t d z
$$

where $u(t)$ is the hazard function given in (2.6) and $g(z)$ the probability density of the shocks $Y$. From this it follows that the process is not Markovian as long as $u(t)$ is not constant, see Jacobsen (2006). Intuitively, for general hazard functions $u(t)$, it is important to know the time elapsed since the last trade and thus the process is not memoryless 1

A special example is the well-known case resulting from the assumption that the waiting times $\tau$ are exponentially distributed with parameter $\lambda$. For this particular case, the survival function is given by $\Upsilon(t)=e^{-\lambda t}$ and the hazard function becomes $u(t)=\lambda$; note that the hazard function is independent of

\footnotetext{
${ }^{1}$ However, if we enlarge the state space with the backward recurrence time $H(t)$, then it can be shown that the twodimensional process $(X(t), H(t))$ is a time-homogeneous Markov process, see Cartea and Meyer-Brandis (2010).
} 
the backward recurrence time $H(t)$. In this case the compensating measure (2.8) becomes $\mathrm{v}(d t, d z)=$ $\lambda g(z) d t d z$, which is the compensating measure of the compound Poisson process $U(t)$.

Finally, the proposition below states that the stock price is an exponential martingale under the risk-neutral measure. The proof relies on the fact that price revisions are such that $\mathbb{E}\left[e^{Y}\right]=1$ and that the predictable compensator of the jump measure $N(d t, d z)$ is given by (2.8).

Proposition 2.1 Let the risk-neutral dynamics of the stock price satisfy SDE (2.3). Assume that $g(z)$ is the density of an infinitely divisible distribution satisfying

$$
\int_{\mathbb{R}}\left(e^{z}-1\right) g(z) d z=0
$$

Then

$$
e^{-(r-D) t} S(t)=S(0) \exp \left(\int_{0}^{t} \int_{\mathbb{R}_{0}} z N(d t, d z)-\int_{0}^{t} \int_{\mathbb{R}_{0}}\left(e^{z}-1\right) v(d t, d z)\right)
$$

is an exponential martingale under $\mathbb{Q}$.

To show the result note that solving SDE (2.3) and discounting it by $e^{-(r-D) t}$ we have that

$$
e^{-(r-D) t} S(t)=S(0) \exp \left(\int_{0}^{t} \int_{\mathbb{R}_{0}} z N(d t, d z)\right)
$$

and, because of condition (2.9), we can rewrite $e^{-(r-D) t} S(t)$ as (2.10).

\section{Waiting-time or duration between trades}

There are a number of studies that have used high-frequency data to study the characteristics of either the waiting-time between trades or the consequences of stochastic trade arrival. For example, Engle (2000) finds evidence that both stock returns and variances are found to be negatively influenced by long durations between trades. The study of Dufour and Engle (2000) shows that the stochastic component of duration can explain the relationship between short time durations, i.e. high trading activity, and both larger quote revisions and stronger positive autocorrelations of trades. Both these findings are 
also supported by the results in Cartea and Meyer-Brandis (2010) where the authors employ a specific survival function of the waiting times known in the literature as the Mittag-Leffler (ML) function given by

$$
\Upsilon_{\mathrm{ml}}(t)=\sum_{j=0}^{\infty}(-1)^{j} \frac{\left(t / \tau_{o}\right)^{\beta j}}{\Gamma(\beta j+1)}, \quad \text { for } 0<\beta \leq 1
$$

and $\tau_{o}>0$ is a constant. One can check that the ML nests the exponential survival function when $\beta=1$.

The ML function was first proposed in a financial context in the work of Mainardi, Raberto, Gorenflo, and Scalas (2000) and Scalas, Gorenflo, and Mainardi (2000). There are two main reasons that make the ML a suitable survival function in a financial context (as well as in many other physical applications). First, the decay of the (right) tail of the waiting-time distribution is algebraic, instead of exponential, something that is observed in a great deal of traded assets and this feature alone makes it a strong candidate to model (unconditional) waiting times between trades. Second, the ML is highly tractable in applications because one can write its Laplace transform in closed-form and because one can express the Laplace-Fourier transform of the MPP $U(t)$ in an analytic form. The latter is possible as long as we know the Fourier transform of the distribution of the random variable $Y$, i.e. the characteristic function, responsible for the marks in the $U(t)$ process.

Although the works cited here present ample evidence supporting the use of the ML survival function, Figure 1 shows a representative example of the shape of the empirical and fitted survival functions for June 2005 trades of General Motors in log-log space. Despite the fact that General Motors is a relatively frequently traded stock, the figure clearly shows that the fitted exponential survival function, (the dashed line), is a very poor assumption when compared to the fitted ML survival function, (solid line). The latter is better at capturing 'longer' periods of time between trades as reflected by the empirical survival function (denoted by circles).

We obtain the probability density function (pdf) of the waiting-time from the ML survival function,

$$
v_{\mathrm{ml}}(t)=-\frac{d}{d t} \Upsilon_{\mathrm{ml}}(t), \quad \text { for } 0<\beta \leq 1
$$




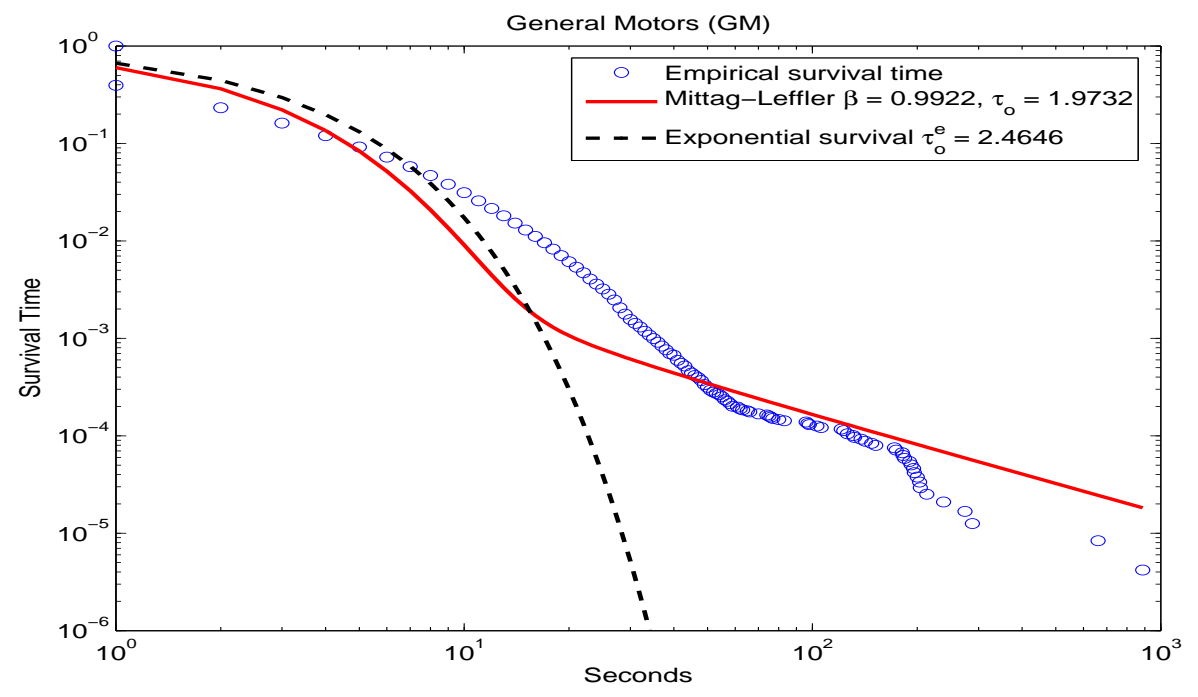

Figure 1. General Motors waiting times: empirical, Mittag-Leffler and Exponential.

and we can also calculate the expected number of trades over the time interval $[0, T]$

$$
\mathbb{E}\left[N(T) \mid \mathcal{F}_{0}\right]=\left(T / \tau_{o}\right)^{\beta} / \Gamma(\beta+1)
$$

The ML arises in many contexts, see for example Podlubny (1999) and Del-Castillo-Negrete, Carreras, and Lynch (2005). In its most general form, the (two-parameter) ML function is given by

$$
E_{\beta, \gamma}(z)=\sum_{j=0}^{\infty} \frac{z^{j}}{\Gamma(\beta j+\gamma)}, \quad \beta>0, \quad \gamma>0
$$

with Laplace transform

$$
\mathcal{L}\left\{t^{\beta n+\gamma-1} E_{\beta, \gamma}^{(n)}\left( \pm a t^{\beta}\right)\right\}=\frac{n ! s^{\beta-\gamma}}{\left(s^{\beta} \mp a\right)^{n+1}}, \quad \operatorname{Re}(s)>|a|^{1 / \gamma},
$$

where $E_{\beta, \gamma}^{(n)}(y)=\frac{d^{n}}{d y^{n}} E_{\beta, \gamma}(y)$ and the Laplace transform of a function $f$ is given by

$$
\mathcal{L}[f(t)]=\tilde{f}(s)=\int_{0}^{\infty} e^{s t} f(t) d t
$$


Below we will also use the Fourier transform of a function $h$ which is denoted by

$$
\hat{h}(\xi)=\mathcal{F}[h(x)]=\int_{-\infty}^{\infty} e^{i x \xi} h(x) d x .
$$

In particular, the survival function we employ here given by equation (3.1) is a one-parameter ML $E_{\beta, 1}\left(t / \tau_{o}\right)$ with Laplace transform

$$
\tilde{\Upsilon}_{\mathrm{ml}}(s)=\frac{1-\tilde{v}_{\mathrm{ml}}(s)}{s}=\tau_{o} \frac{\left(\tau_{o} s\right)^{\beta-1}}{1+\left(\tau_{o} s\right)^{\beta}}, \quad \text { where } \quad \tilde{v}_{\mathrm{ml}}(s)=\mathcal{L}\left\{v_{\mathrm{ml}}(t)\right\}, \quad \text { for } 0<\beta \leq 1
$$

We end this section by presenting an infinite order approximation of the function $E_{\beta, 1}\left[a\left(e^{z}-1\right)\right]$ where $a$ is a constant. Expanding the exponential function $e^{z}=1+z+\frac{1}{2} z^{2}+\cdots$ allows us to write

$$
\begin{aligned}
E_{\beta, 1}\left[a\left(e^{z}-1\right)\right]= & 1+\frac{a\left(e^{z}-1\right)}{\Gamma(\beta+1)}+\frac{a^{2}\left(e^{z}-1\right)^{2}}{\Gamma(2 \beta+1)}+\frac{a^{3}\left(e^{z}-1\right)^{3}}{\Gamma(3 \beta+1)}+\cdots \\
= & 1+\frac{a}{\Gamma(\beta+1)}\left(z+\frac{1}{2} z^{2}+\cdots\right)+\frac{a^{2}}{\Gamma(2 \beta+1)}\left(z+\frac{1}{2} z^{2}+\cdots\right)^{2} \\
& +\frac{a^{3}}{\Gamma(3 \beta+1)}\left(z+\frac{1}{2} z^{2}+\cdots\right)^{3}+\cdots \\
= & 1+\frac{a}{\Gamma(\beta+1)} z+\cdots+\frac{a^{2}}{\Gamma(2 \beta+1)} z^{2}+\cdots+\frac{a^{3}}{\Gamma(3 \beta+1)} z^{3}+\cdots \\
= & E_{\beta, 1}[a z]+\cdots .
\end{aligned}
$$

\section{PIDE with non-local operator in time-to-maturity}

In this section I derive the main result of this paper. I do this in 3 steps. First, Proposition 4.1 shows that we can express the Laplace-Fourier transform of the MPP that drives the marks and arrival of trades in our model in closed-form. Second, Proposition 4.2 shows that for a general European-style option we can write the solution to the pricing problem as a numerical inversion of the Fourier transform of the value of the option where, as expected, knowledge of the Fourier transform of the MPP $U(t)$ is required. Third, Proposition 4.3 contains the main result where I show that the PIDE satisfied by European-style options contains a non-local operator in time-to-maturity. 
Moreover, Proposition 4.4 shows the bounds satisfied by vanilla put and call options when the time since the last trade is $H(t)>0$. And finally, before showing numerical examples in Section 5 I discuss some features of the pricing PIDE derived in Proposition 4.3. I point out the connection between the non-explosive MPP that we employ here to model prices. I also show that the infinitesimal operator which appears in the pricing PIDE when $\beta=1$, coincides with the infinitesimal operator of a Lévy process which appears in the PIDE for exponential Lévy processes of either finite or infinite activity.

Proposition 4.1 Let

$$
q(x, 0, T)=e^{U(T)}
$$

where

$$
U(T)=\sum_{n=1}^{N(T)} Y_{i}
$$

is an MPP as described above in equation (2.4) where $v(t)$ is the waiting-time pdf. Assume that the backward recurrence time $H(0)=0$, i.e. a trade just happened. Then

$$
\mathcal{L}\{\mathcal{F}\{q(x, 0, T)\}\}=\frac{1-\tilde{v}(s)}{s} \frac{1}{1-e^{\Psi(\xi)} \tilde{v}(s)},
$$

where $e^{\Psi(\xi)}$ is the characteristic function of $Y$ with $\Psi(\xi)$ given by 1.1 .

Proof. Let $P(n, T)$ denote the probability density function of observing $n$ trades during the time interval $[0, T]$. Using the survival function $(2.5)$, the probability that a trade does not take place before time $T$, and the fact that we have assumed that $H(0)=0$, is given by

$$
P(n=1, T)=\int_{0}^{T} v(s) \Upsilon(T-s) d s=(\mathrm{v} \star \Upsilon)(T),
$$

where $\star$ denotes convolution. Then the probability of observing $n$ trades over the interval $[0, T]$ is given by $\left(v^{n} \star \Upsilon\right)(T)$ and taking its Laplace transform yields

$$
\tilde{P}(n, s)=(\tilde{v}(s))^{n} \tilde{\Upsilon}(s)=(\tilde{v}(s))^{n} \frac{1-\tilde{v}(s)}{s}
$$


Now we calculate

$$
\begin{aligned}
\hat{q}(\xi, 0, T) & =\mathbb{E}\left[e^{i \xi \sum_{i=1}^{N(T)} Y_{i}}\right] \\
\mathcal{L}\{\hat{q}(\xi, 0, T)\} & =\mathcal{L}\left\{\mathbb{E}\left[e^{N(T) \Psi(\xi)}\right]\right\} \\
& =\mathcal{L}\left\{\sum_{0}^{\infty} P(n, T) e^{n \Psi(\xi)}\right\} \\
& =\frac{1-\tilde{v}(s)}{s} \frac{1}{1-e^{\Psi(\xi)} \tilde{v}(s)},
\end{aligned}
$$

as required.

Proposition 4.2 Let $G(\cdot)$ be the pay-off function of a European-style option with maturity $T$ written on the stock $S(t)$ that follows the risk-neutral log-stock price process

$$
d X(t)=(r-D) d t+Y d N(t)
$$

where $X_{t}=\ln S(t), N(t)$ is a counting process with survival function $\Upsilon(t), r$ is the risk-free rate and $D$ is a continuous dividend yield. Let the price revisions $Y$ be i.i.d. Lévy distributed with triplet $\left(w, \sigma^{2}, W\right)$ and characteristic exponent $\Psi(\xi)$ with $w$ a convexity adjustment so that $\mathbb{E}\left[e^{-r(T-t)} S(T) \mid \mathcal{F}_{t}\right]=e^{-D(T-t)} S(t)$, where $\mathbb{E}\left[\cdot \mid \mathcal{F}_{t}\right]$ is the expectation operator conditioned on information at time $t$.

Assume that $\hat{q}(\xi, t, T)$, defined by

$$
\hat{q}(\xi, t, T):=\mathbb{E}\left[e^{i \xi \sum_{i=N(t)+1}^{N(T)} Y_{i}} \mid \mathcal{F}_{t}\right]
$$

is analytic in $\xi$ in a strip that intersects the strip where the (complex) Fourier transform of $G$ exists. Let $\hat{\xi} \in \mathbb{R}$ be such that the line $[-\infty+i \hat{\xi}, \infty+i \hat{\xi}]$ is part of this intersection. Then, if $H(0)$ (i.e. the time elapsed since the last trade is zero) the price at time t of the European option with pay-off $G(\cdot)$, and maturity $T$, is given by

$$
V(x, t ; T)=\frac{e^{-r(T-t)}}{2 \pi} \int_{-\infty+i \hat{\xi}}^{\infty+i \hat{\xi}} e^{-i \xi \ln S(t)} e^{-i \xi(r-D)(T-t)} \hat{q}(-\xi, t, T) \hat{G}(\xi) d \xi
$$


Proof. Assuming the pay-off $G(\cdot)$ is such that we can invert its Fourier transform,

$$
\begin{aligned}
V(x, t ; T) & =e^{-r(T-t)} \mathbb{E}\left[G(X(T)) \mid \mathcal{F}_{t}\right] \\
& =e^{-r(T-t)} \mathbb{E}\left[\frac{1}{2 \pi} \int_{-\infty+i \xi_{i}}^{\infty+i \xi_{i}} e^{-i \xi x(T)} \hat{G}(\xi) d \xi \mid \mathcal{F}_{t}\right] \\
& =\frac{e^{-r(T-t)}}{2 \pi} \int_{-\infty+i \xi_{i}}^{\infty+i \xi_{i}} e^{-i \xi \ln S(t)} e^{-i \xi(r-D)(T-t)} \mathbb{E}\left[e^{-i \xi \sum_{i=1}^{N(T)} Y_{i}} \mid \mathcal{F}_{t}\right] \hat{G}(\xi) d \xi \\
& =\frac{e^{-r(T-t)}}{2 \pi} \int_{-\infty+i \xi_{i}}^{\infty+i \xi_{i}} e^{-i \xi \ln S(t)} e^{-i \xi(r-D)(T-t)} \hat{q}(-\xi, t, T) \hat{G}(\xi) d \xi .
\end{aligned}
$$

Note that in Proposition 4.2 I have not specified a particular survival function.

Pricing European-style options when stock prices follow an exponential Lévy process can be done by either solving PIDE (2.2) or by employing Fourier methods. In the latter case we would proceed in the same way as in Proposition 4.2, with $S(t)$ given by (2.1), and evaluate the Fourier inversion

$$
V(S, t ; K, T)=-\frac{e^{-r(T-t)} K}{2 \pi} \int_{-\infty+i \hat{\xi}}^{\infty+i \hat{\xi}} e^{-i \xi \ln S(t)+(T-t)[-i \xi(r-D)+\Psi(-\xi)]} \hat{G}(\xi) d \xi
$$

where $\Psi(\xi)$ is given by the Lévy-Khintchine formula (1.1).

The main difference between equations (4.5) and (4.6) is that in the former we need the characteristic function of the MPP that drives the arrival and sizes of price revisions, in other words we need to know $\hat{q}(\xi, t, T)$ or be able to perform a numerical inversion to calculate it, and in the latter we need (1.1).

If in Proposition 4.2 we let the survival function be given by (3.1), then we know $\hat{q}$ in closed-form. Furthermore, if we let $\beta=1$ in 3.1 , we can verify that the price of a European call option with strike $K$ and maturity $T$ is given by 2

$$
V(S, t ; K, T)=-\frac{e^{-r(T-t)} K}{2 \pi} \int_{-\infty+i \hat{\xi}}^{\infty+i \hat{\xi}} e^{-i \xi \ln S(t)+(T-t)\left[-i \xi(r-D)+(\Psi(-\xi)-1) \tau_{o}^{-1}\right]} \frac{K^{i \xi}}{\xi^{2}-i \xi} d \xi
$$

for $\hat{\xi}>1$.

\footnotetext{
${ }^{2}$ Note that we must require $e^{\Psi(-\xi)}$ to be analytic in a line that intersects $[-\infty+i \hat{\xi}, \infty+i \hat{\xi}]$ where $\hat{\xi}>1$.
} 
When $\beta<1$ we know that the MPP in the price dynamics (4.3) is not Markovian. This nonMarkovian characteristic of the underlying stock shows up in the PIDE satisfied by financial derivatives written on $S(t)$ in the form of a non-local operator. In particular, the non-local operator we obtain here is known as the Caputo (fractional derivative in time) which, for a function $f$, is defined as

$$
{ }_{0}^{c} D_{T}^{\beta} f(T)=\frac{1}{\Gamma(1-\beta)} \int_{0}^{T} \frac{\partial f(u) / \partial u}{(T-u)^{\beta}} d u
$$

with Laplace transform

$$
\mathcal{L}\left\{{ }_{0}^{c} D_{T}^{\beta} f(T)\right\}=s^{\beta} \tilde{f}(s)-s^{\beta-1} f(0),
$$

see Podlubny (1999).

Proposition 4.3 Let $G(\cdot)$ be the pay-off function of a European-style option with maturity $T$ written on the stock $S(t)$ that follows the risk-neutral log-stock price process (4.3) in Proposition 4.2 with ML survival function given by (3.1). Assume that $\hat{q}(\xi, t, T)$, defined by

$$
\hat{q}(\xi, t, T):=\mathbb{E}\left[e^{i \xi \sum_{i=N(t)+1}^{N(T)} Y_{i}} \mid \mathcal{F}_{t}\right]
$$

is analytic in $\xi$ in a strip that intersects the strip where the (complex) Fourier transform of $G$ exists. Let $\hat{\xi} \in \mathbb{R}$ be such that the line $[-\infty+i \hat{\xi}, \infty+i \hat{\xi}]$ is part of this intersection. Then, if $H(0)$ (i.e. the time elapsed since the last trade is zero) and using the approximation of the ML function discussed above in (3.6):

$$
E_{\beta, 1}\left[e^{z}-1\right] \sim E_{\beta, 1}[z]
$$

the price at time $t$ of the European option with pay-off $G(\cdot)$, and maturity $T$, satisfies the following PIDE, in time-to-maturity,

$$
\begin{aligned}
{ }_{0}^{c} D_{T}^{\beta} e^{r T} V(\bar{x}, T)= & \tau_{o}^{-\beta} e^{r T}\left(w \frac{\partial V(\bar{x}, T)}{\partial \bar{x}}+\frac{1}{2} \sigma^{2} \frac{\partial^{2} V(\bar{x}, T)}{\partial \bar{x}^{2}}\right) \\
& +\tau_{o}^{-\beta} e^{r T} \int_{\mathbb{R}_{0}}\left[V(\bar{x}+y, T)-V(\bar{x}, T)-u(y) \frac{\partial V(\bar{x}, T)}{\partial \bar{x}}\right] W(d y),
\end{aligned}
$$


where $\bar{x}=x-(r-D) T$.

Proof. Without loss of generality let $t=0$. Using Proposition 4.1 and (3.4) we write equation (4.9) as

$$
E_{\beta, 1}\left[\left(e^{\Psi(\xi)}-1\right)\left(T / \tau_{o}\right)^{\beta}\right]
$$

and using (4.10) we obtain

$$
\hat{q}(-\xi, 0, T)=E_{\beta, 1}\left[\Psi(-\xi)\left(T / \tau_{o}\right)^{\beta}\right]
$$

From Proposition 4.2 we can write

$$
\begin{aligned}
\hat{V}(\xi, T) & =e^{-r T} e^{-i \xi \ln S(0)} e^{-i \xi(r-D) T} E_{\beta, 1}\left[\Psi(-\xi)\left(T / \tau_{o}\right)^{\beta}\right] \hat{G}(\xi), \\
\frac{\hat{V}(\xi, T) e^{i \xi \ln S(0)}}{\hat{G}(\xi)} & =e^{-(r+i \xi(r-D)) T} E_{\beta, 1}\left[\Psi(-\xi)\left(T / \tau_{o}\right)^{\beta}\right] \\
\mathcal{L}\left\{\frac{\hat{V}(\xi, T) e^{i \xi \ln S(0)}}{\hat{G}(\xi)}\right\} & =\frac{u^{\beta-1}}{u^{\beta}-\Psi(-\xi) \tau_{o}^{-\beta}}, \quad \text { using (3.4) } \\
\tilde{\hat{I}}(\xi, s) & =\frac{u^{\beta-1}}{u^{\beta}-\Psi(-\xi) \tau_{o}^{-\beta}},
\end{aligned}
$$

where for notational simplicity

$$
\hat{I}(\xi, T)=\frac{\hat{V}(\xi, T) e^{i \xi \ln S(0)}}{\hat{G}(\xi)} \quad \text { and } \quad u=s-(r+i \xi(r-D)) T
$$

Proceed by rearranging equation (4.13) to obtain

$$
u^{\beta} \tilde{\hat{I}}(\xi, s)-u^{\beta-1}=\Psi(-\xi) \tau_{o}^{-\beta} \tilde{\hat{I}}(\xi, s) .
$$

Substituting $T=0$ in (4.12) shows that $\hat{I}(\xi, 0)=1$ and using (4.8), we have that

$$
\mathcal{L}\left\{e^{-(r+i \xi(r-D)) T}{ }_{0}^{c} D_{T}^{\beta} e^{(r+i \xi(r-D)) T} \hat{I}(\xi, T)\right\}=u^{\beta} \tilde{\hat{I}}(\xi, s)-u^{\beta-1} .
$$


Therefore using equations (4.14) and (4.15) we obtain

$$
\begin{aligned}
e^{-(r+i \xi(r-D)) T}{ }_{0}^{c} D_{T}^{\beta} e^{(r+i \xi(r-D)) T} \hat{I}(\xi, T) & =\Psi(-\xi) \tau_{o}^{-\beta} \hat{I}(\xi, T) \\
{ }_{0}^{c} D_{T}^{\beta} e^{(r+i \xi(r-D)) T} \hat{V}(\xi, T) & =\Psi(-\xi) \tau_{o}^{-\beta} e^{(r+i \xi(r-D)) T} \hat{V}(\xi, T) .
\end{aligned}
$$

Now the final step is to take the inverse Fourier transform of (4.16) to obtain (4.11) as required.

Above, I made the simplifying assumption that the time elapsed since the last trade was zero, $H(t)=0$. However, the price of a financial instrument with terminal payoff $G(X(T))$ and $H(t)>0$ is given by

$$
V(x, t)=e^{-r(T-t)} \mathbb{E}[G(X(T)) \mid X(t), H(t)]=\left.e^{-r(T-t)} \mathbb{E}^{x}\left[G\left(X^{h}(T-t)\right)\right]\right|_{x=X(t), h=H(t)},
$$

where $X^{h}(t)$ is the $h$-delayed renewal process starting in $x$, induced by $X(t)$, i.e. the first waiting-time in (2.3) has the distribution of $\left(\tau_{1}-h\right)$, given $\tau_{1}>h$.

Calculating prices when $H(t)>0$ using (4.17) might be slightly involved. Depending on the model employed, and the type of instrument we are pricing, it might be better to approximate the price $V(x, t)=e^{-r(T-t)} \mathbb{E}[G(X(T)) \mid X(t), H(t)]$ with $V(x, t)=e^{-r(T-t)} \mathbb{E}[G(X(T)) \mid X(t), H(t)=0]$. The Proposition below shows that we can obtain upper and lower bounds for European puts and calls when $H(t)>0$.

Proposition 4.4 Let $H(t)>0$. Let $C(x, t ; T, H(t))$ denote the price of a European call and $P(x, t ; T, H(t))$ the price of a European put struck at $K$ with maturity $T$ written on the stock $S(t)$ that follows the riskneutral log-stock price process (4.3) in Proposition 4.2 with survival function $\Upsilon(t)$. Assume that the hazard function, see (2.6), is increasing in $t$. Then $C(x, t ; T, H(t))$ obeys the bounds

$$
C(x, t ; T, 0) \leq C(x, t ; T, H(t)) \leq \mathbb{E}[C(x+Y, t ; T, 0)]
$$


and $P(x, t ; T, H(t))$ obeys the bounds

$$
P(x, t ; T, 0) \leq P(x, t ; T, H(t)) \leq \mathbb{E}[P(x+Y, t ; T, 0)] .
$$

Proof. We know that

$$
C\left(x, t ; T_{1}, H(t)\right) \leq C\left(x, t ; T_{2}, H(t)\right),
$$

for $T_{2}-t \geq T_{1}-t$. Let $\tau_{h}$ denote the time of the next arrival of a trade given that you have waited an amount $h$ already. Then $\tau_{0} \geq \tau_{H(t)}$ a.s. and clearly

$$
C(x, t ; T, H(t))=\mathbb{E}\left[C\left(x+Y, \tau_{H(t)} ; T, 0\right)\right],
$$

i.e. wait until next trade, then $x$ jumps by $Y$, and waiting-time resets to 0 . Also,

$$
C(x, t ; T, 0)=\mathbb{E}\left[C\left(x+Y, \tau_{0} ; T, 0\right)\right],
$$

i.e. wait until next trade, then $x$ jumps by $Y$, and waiting-time resets to 0 .

Lower Bound. Using inequality (4.20) we have $C\left(x, \tau_{0} ; T, 0\right) \leq C\left(x, \tau_{H(t)} ; T, 0\right)$ for every $x$ a.s. since $T-\tau_{H(t)} \geq T-\tau_{0}$ a.s.. Consequently,

$$
\mathbb{E}\left[C\left(x+Y, \tau_{0} ; T, 0\right)\right] \leq \mathbb{E}\left[C\left(x+Y, \tau_{H(t)} ; T, 0\right],\right.
$$

where expectation is over both $Y$ and the random trade times. Now, the left-hand side of (4.23) is equal to $C(x, t ; T, 0)$, see (4.22), and the right-hand side is $C(x, t ; T, H(t))$, see (4.21).

Upper Bound. Using (4.20) we know that $C(x, 0 ; T, 0) \geq C\left(x, \tau_{H(t)} ; T, 0\right)$, for every $x$ a.s. and since $\tau_{H(t)} \geq 0$ a.s., we have that

$$
E\left[C(x+Y, 0 ; T, 0) \geq \mathbb{E}\left[C\left(x+Y, \tau_{H(t)} ; T, 0\right)\right]=C(x, t ; T, H(t)),\right.
$$

where expectation is over both $Y$ and the random trade times. 
The proof for the put option is similar.

\subsection{Obtaining the infinitesimal generator of a Lévy process from a non-explosive MPP}

Before proceeding to the next section, where we price European vanilla options using the result in Proposition 4.3 and calculate upper and lower bounds for option prices when $H(t)>0$, I comment on the particular case when $\beta=1$ and discuss the connection between (4.11) and the traditional PIDE (2.2) that arises in exponential Lévy processes.

First, it is trivial to see that when $\beta=1$ equation (4.11) becomes

$$
\begin{aligned}
\frac{d}{d T} e^{r T} V(\bar{x}, T)= & \tau_{o}^{-\beta} e^{r T}\left(w \frac{\partial V(\bar{x}, T)}{\partial \bar{x}}+\frac{1}{2} \sigma^{2} \frac{\partial^{2} V(\bar{x}, T)}{\partial \bar{x}^{2}}\right) \\
& +\tau_{o}^{-\beta} e^{r T} \int_{\mathbb{R}_{0}}\left[V(\bar{x}+y, T)-V(\bar{x}, T)-u(y) \frac{\partial V(\bar{x}, T)}{\partial \bar{x}}\right] W(d y), \\
r V(x, T)+\frac{\partial V(x, T)}{\partial T}= & \left(r-D-\tau_{o}^{-\beta} w\right) \frac{\partial V(x, T)}{\partial x} \\
& +\tau_{o}^{-\beta} \frac{1}{2} \sigma^{2} \frac{\partial^{2} V(x, T)}{\partial x^{2}} \\
& +\int_{\mathbb{R}_{0}}\left[V(x+y, T)-V(x, T)-u(y) \frac{\partial V(x, T)}{\partial x}\right] \tau_{o}^{-\beta} W(d y),
\end{aligned}
$$

which is the usual PIDE in time-to-maturity when the underlying asset follows an exponential Lévy process.

If we let $c=\tau_{o}^{-\beta} w, W_{o}=\tau_{o}^{-\beta} W$ and $\sigma_{o}^{2}=\tau_{o}^{-\beta} \sigma^{2}$ in (4.24) we obtain the traditional PIDE (2.2) for the general family of exponential Lévy models, with triplet $\left(0, \sigma_{o}^{2}, W_{o}\right)$ and convexity adjustment $c$, which includes both finite and infinite activity models. This result is interesting because the model is based on a non-explosive MPP, i.e. there are a finite number of trades in a finite time interval, but using (4.10) the resulting PIDE with $\beta=1$ is equivalent to having assumed exponential Lévy processes of infinite activity when $\Psi(\xi)$ is the characteristic exponent of an infinite activity Lévy process at $t=1$.

Moreover, I further note that using the approximation (4.10) does not imply that the MPP becomes an explosive MPP with an infinite arrival of trades over a finite time interval. What I have shown is that 
the distribution of the MPP using (4.10) can be the same as that of a Lévy process of infinite activity (depending on the Lévy measure of the price revisions $Y$ ). Thus, the result is about the distributional properties of the MPP, not about its path properties.

\section{Forward Fokker-Planck equation}

It is also interesting to inspect the result that we obtain when we use (4.10) by looking at the Forward Fokker-Planck (FFP) equation of the MPP $U(t)$. Assume for simplicity that $r=D=0$, so the stock price is $S(T)=S(0) e^{U(T)}$ where

$$
U(T)=\sum_{n=1}^{N(T)} Y_{i}
$$

Assume that $p(x, T)$ is the probability that at time $T$ the $\log$-price, starting at $x(0)=\ln S(0)$, is at $x(T)$. To derive the FFP we first derive the transition density satisfied by $p(x, T)$.

Recall that the pdf of the waiting time $\tau_{n}=T_{n}-T_{n-1}, n=1,2,3, \ldots$, between trades is denoted by $v(\tau)$. The probability that the $\log$-price is $x(T)$ at a future time $T$, is given by

$$
p(x, T)=\delta(x) \int_{T}^{\infty} v(t) d t+\int_{0}^{T} v(T-t)\left[\int_{-\infty}^{\infty} g\left(x-x^{\prime}\right) p\left(x^{\prime}, t\right) d x^{\prime}\right] d t
$$

where $\delta(x)$ is the delta function. The first term on the right-hand side accounts for no trades arriving over the interval $(0, T]$, so the log-price remains unchanged at $x(0)$. The second term denotes the contribution that a movement in the underlying stock, located at $x^{\prime}$ and which jumps to $x(T)$ during $(0, T]$, makes to $p$.

Now I derive the FFP equation satisfied by $p(x, T)$. First solve (4.26) by taking Fourier-Laplace transforms:

$$
\hat{\tilde{p}}(s, \xi)=\frac{1-\tilde{v}(s)}{s} \frac{1}{1-\tilde{v}(s) e^{\Psi(\xi)}}
$$


and recall that $e^{\Psi(\xi)}$ is the characteristic function of $g$; the distribution of the i.i.d. Lévy distributed jumps $Y$ with triplet $\left(w, \sigma^{2}, W\right)$. Then if we assume that the waiting-time distribution is that of the ML survival function (3.4), with $a=1$ for simplicity, and use the approximation (4.10) we obtain

$$
\hat{\tilde{p}}(\xi, s)=\frac{s^{\beta-1}}{s^{\beta}-\Psi(\xi)}, \quad \text { for } 0<\beta \leq 1
$$

Proceeding in a similar way as in the proof of Proposition 4.2, we have

$$
\begin{array}{rlr}
\hat{\tilde{p}}(\xi, s) s^{\beta}-s^{\beta-1} & =\Psi(\xi) \hat{\tilde{p}}(\xi, s) & \\
\tilde{p}(x, s) s^{\beta}-s^{\beta-1} \delta(x) & =\mathrm{L} \tilde{p}(x, s), & \text { by taking } \mathcal{F}^{-1}, \\
{ }_{0}^{c} D_{T}^{\beta} p(x, T) & =\mathrm{L} p(x, T), & \text { by taking } \mathcal{L}^{-1},
\end{array}
$$

where $\mathrm{L}$ is the infinitesimal generator of the Lévy process, with triplet $\left(w, \sigma^{2}, W\right)$, given by

$$
\begin{aligned}
\operatorname{L} p(x, T)= & \frac{1}{2 \pi} \int_{-\infty}^{\infty} e^{-i \xi x} \Psi(\xi) \hat{p}(\xi, T) d \xi \\
= & -w \frac{\partial p(x, T)}{\partial x}+\frac{1}{2} \sigma^{2} \frac{\partial^{2} p(x, T)}{\partial x^{2}} \\
& +\int_{-\infty}^{\infty}\left(p(x-y, T)-p(x, T)+u(y) \frac{\partial p(x, T)}{\partial x}\right) W(d y) .
\end{aligned}
$$

Hence, $p(x, T)$ satisfies the FFP equation

$$
\begin{aligned}
{ }_{0}^{c} D_{T}^{\beta} p(x, T)= & -w \frac{\partial p(x, T)}{\partial x}+\frac{1}{2} \sigma^{2} \frac{\partial^{2} p(x, T)}{\partial x^{2}} \\
& +\int_{\mathbb{R}_{0}}\left(p(x-y, T)-p(x, T)+u(y) \frac{\partial p(x, T)}{\partial x}\right) W(d y) .
\end{aligned}
$$

We see that for $\beta=1$ we obtain

$$
\begin{aligned}
\frac{\partial p(x, T)}{\partial T}= & -w \frac{\partial p(x, T)}{\partial x}+\frac{1}{2} \sigma^{2} \frac{\partial^{2} p(x, T)}{\partial x^{2}} \\
& +\int_{\mathbb{R}_{0}}\left(p(x-y, T)-p(x, T)+u(y) \frac{\partial p(x, T)}{\partial x}\right) W(d y)
\end{aligned}
$$

which is the well-known FFP equation for Lévy processes and, depending on the Lévy measure $W(d y)$, is of either finite or infinite activity. 
Finally, note that the result that links the distribution of a non-explosive MPP to the distribution of a Lévy process of infinite activity can be obtained by starting with a compound Poisson process $\sum_{n=1}^{N(t)} Y_{i}$ where $N(t)$ is a homogeneous Poisson process with intensity $\lambda t$, and $Y_{i}$ are i.i.d. with infinitely divisible distribution and triplet $\left(a, \sigma^{2}, W\right)$. The characteristic function of the compound Poisson process is

$$
\mathbb{E}\left[e^{i \xi \sum_{n=1}^{N(t)} Y_{i}}\right]=e^{\lambda t\left(e^{\Psi(\xi)}-1\right)}
$$

and using (4.10) with $\beta=1$, i.e. $e^{\Psi(\xi)} \sim 1+\Psi(\xi)$, we can write

$$
\begin{aligned}
\ln \mathbb{E}\left[e^{i \xi \sum_{n=1}^{N(t)} Y_{i}}\right] & \sim \lambda t \Psi(\xi) \\
& =\lambda a i \xi t-\frac{1}{2} \lambda \sigma^{2} \xi^{2} t+t \int_{\mathbb{R}_{0}}\left(e^{i \xi y}-1-i \xi u(y)\right) \lambda W(d y),
\end{aligned}
$$

which is the characteristic function of a Lévy process with triplet $\left(\lambda a, \lambda \sigma^{2}, \lambda W\right)$.

\section{Numerical examples: the impact of waiting times on option prices}

To price European-style options we can proceed by either numerically solving PIDE (4.11) or we can also use Fourier methods to invert (4.5) with

$$
\hat{q}(-\xi, t, T)=E_{\beta, 1}\left[\Psi(-\xi)\left(T / \tau_{o}\right)^{\beta}\right]
$$

The advantage of having PIDE (4.11) is that we can solve other problems that involve more exotic payoffs such as knock-out options. There are a number of numerical methods to solve PIDEs that involve non-local Caputo operators, or other fractional derivatives such as Riemann-Liouville, that can be employed to solve (4.11), see Podlubny (1999), and in the context of option pricing with RiemannLiouville fractional operators see Cartea and del Castillo-Negrete (2007).

The numerical examples shown below are prices of European call options which were calculated via Fourier methods using (4.12). The first example assumes that price revisions are Gaussian and the second example assumes that price revisions possess a CGMY distribution (see Carr, Geman, 
Madan, and Yor (2002)). All examples assume that $\tau_{o}=1 / 1,200,000$, (i.e. that there are, on average, $100,000^{\beta} / \Gamma(\beta+1)$ trades per month, see (3.2)).

\section{Gaussian price revisions and ML waiting-times}

Figure 2 shows implied volatility (IV) calculated from the prices of European call options, with $S(0)=$ 100 and $K \in[92,108]$, when it is assumed that price revisions $Y$ are Gaussian with volatility $\sigma=0.5 \sqrt{\tau_{o}}$ and mean $-\sigma^{2} / 2$ (so that $\mathbb{E}\left[e^{Y}\right]=1$ ). With this choice of volatility, and letting $\beta=1$, the model is asymptotically equivalent to assuming a Black-Scholes model with volatility $\sigma_{b s}=0.50$. The Figure shows IVs for $\beta=0.75$. Note that the waiting-time affects the convexity of the IV in a symmetric way and does not reproduce smirks or skewed IVs.

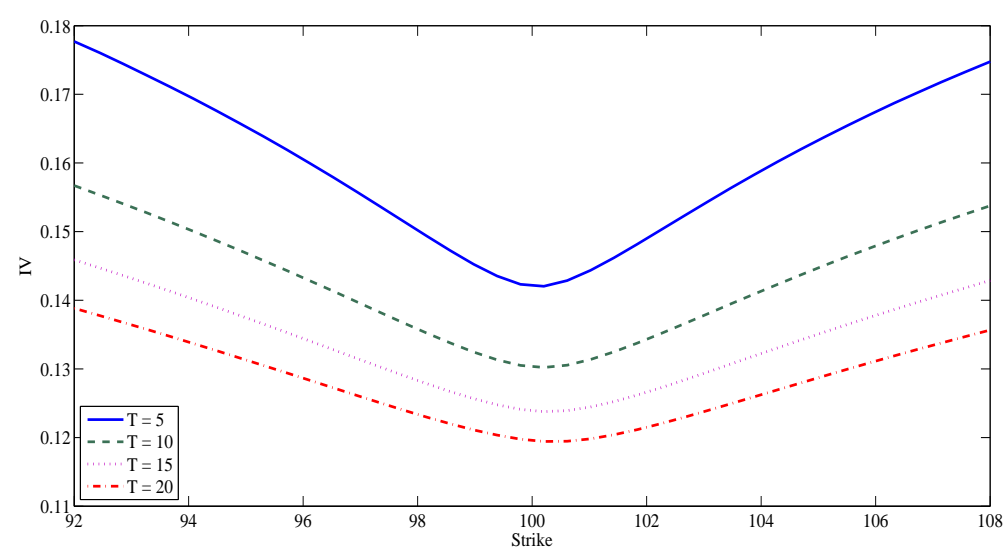

Figure 2. IV across strikes for conditionally Gaussian model with waiting times for different days to maturity $T=$ $\{20,15,10,5\}$ and $\beta=0.75$. The distribution of the price revisions $Y$ is Gaussian, $Y \sim N\left(-\sigma^{2} / 2, \sigma^{2}\right)$ where $\sigma=0.5 \sqrt{\tau_{o}}$, $\tau_{o}=1 /(1,200,000)$ and the parameters for option pricing are $r=5 \%, D=0$ and $S(0)=100$.

\section{CGMY price revisions and ML waiting-times}

Here I show the same results as above, but allow the distribution of price revisions to exhibit fatter tails than the Gaussian distribution by choosing price revisions with a CGMY distribution. The examples assume that $C=1.8750 \times 10^{-7}, Y=1.5, G=10, M=20$, which implies that the distribution of the price shocks has negative asymmetry because $G<M$, and both the left and right tails of the distribution of price shocks are heavier than those of a Normal distribution. 
Figure 3 shows IVs across strikes and maturities for $\beta=0.75$. In this case we observe that the IVs are more pronounced, as well as skewed, than those observed in the Gaussian case; a finding more in line with what we observe in the financial markets.

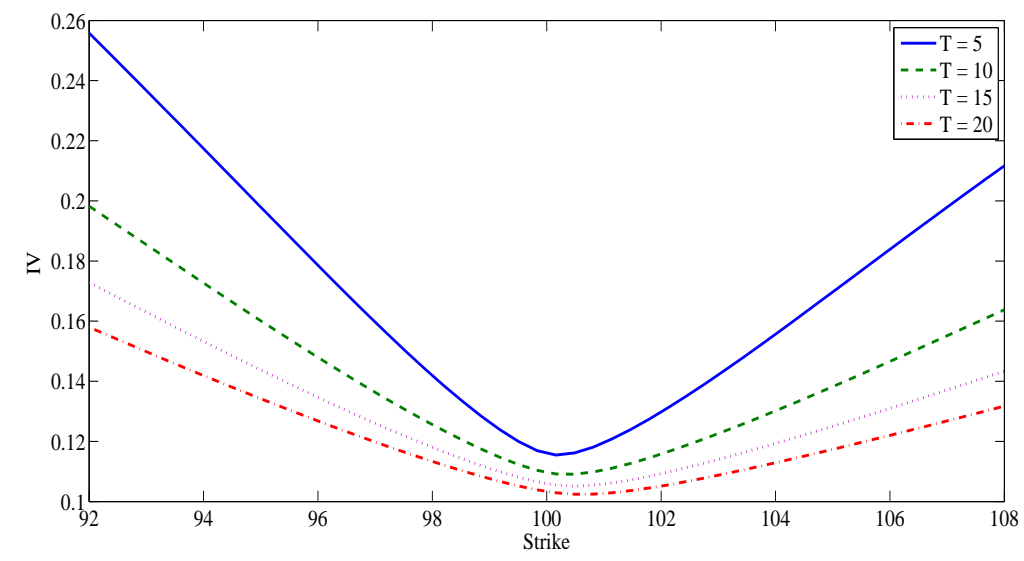

Figure 3. IV across strikes for conditionally CGMY model with waiting times for different days to maturity $T=$ $\{20,15,10,5\}$ and $\beta=0.75$. Here $C=1.8750 \times 10^{-7}, Y=1.5, G=10, M=20, \tau_{o}=1 /(1,200,000)$ and the parameters for option pricing are $r=5 \%, D=0$ and $S(0)=100$.

Price bounds when $H(t)>0$

Figures 4 and 5 depict the difference between the upper and lower bound for the conditionally Gaussian and CGMY prices presented in Figures 2 and 3 . As discussed above, when $H(t)>0$ calculating option prices becomes more involved for general waiting-time distributions. Figures 4 and 5 are calculated using the same parameters as above and we can see that the difference between the two bounds is negligible if we were to compare it to bid/ask spreads observed in the market or if we compare it to tick sizes. This result should not be surprising in this model because the MPP we employ assumes that the expected number of trades is very large. Recall that in our examples with the ML survival function the expected number of trades is $100,000^{\beta} / \Gamma(\beta+1)$ per month, and the bounds are calculated assuming one fewer trade (lower bound) or one extra trade (upper bound) than the number of trades that take place between $t$ and $T$ when $H(t)>0$. 


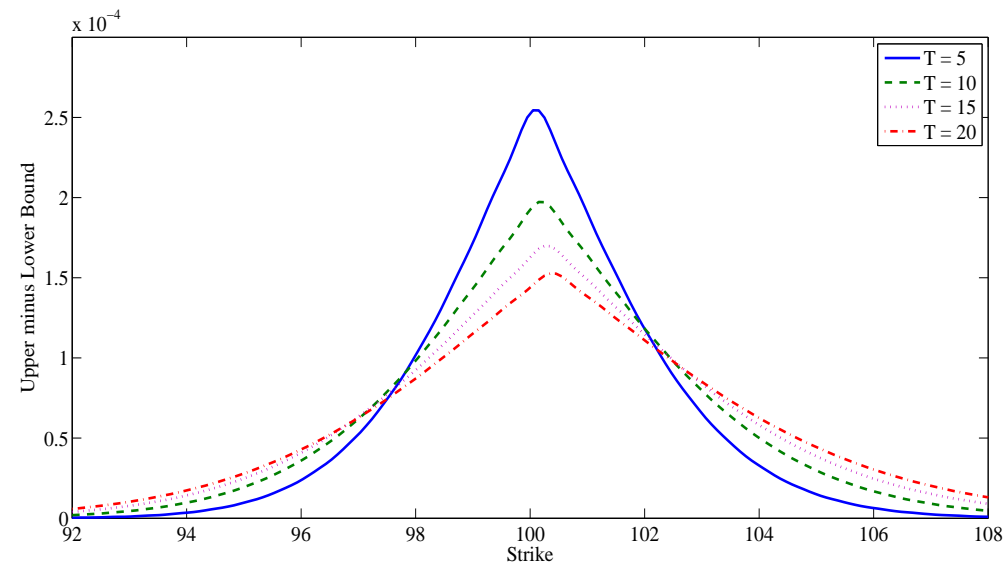

Figure 4. Difference between upper and lower bound from Proposition 4.4 across strikes in the conditionally Gaussian model for different days to maturity $T=\{20,15,10,5\}$ and $\beta=0.75$. The distribution of the price revisions is $Y \sim N\left(-\sigma^{2} / 2, \sigma^{2}\right)$ where $\sigma=0.5 \sqrt{\tau_{o}}, \tau_{o}=1 /(1,200,000)$ and the parameters for option pricing are $r=5 \%, D=0$ and $S(0)=100$.

\subsection{How good is the approximation $E_{\beta, 1}\left[a\left(e^{z}-1\right)\right] \sim E_{\beta, 1}[a z]$ when pricing options?}

In this subsection I discuss the validity of using the approximation

$$
E_{\beta, 1}\left[\left(e^{\Psi(\xi)}-1\right)\left(T / \tau_{o}\right)^{\beta}\right] \sim E_{\beta, 1}\left[\Psi(\xi)\left(T / \tau_{o}\right)^{\beta}\right]
$$

when calculating option prices. We can compare the difference in option prices by employing (4.5) with

$$
\hat{q}(-\xi, t, T)=E_{\beta, 1}\left[\left(e^{\Psi(-\xi)}-1\right)\left(T / \tau_{o}\right)^{\beta}\right]
$$

or

$$
\hat{q}(-\xi, t, T)=E_{\beta, 1}\left[\Psi(-\xi)\left(T / \tau_{o}\right)^{\beta}\right]
$$

Figure 6 shows the difference between call options employing (5.1) and call options employing (5.2), assuming that price revisions are CGMY distributed with $T=\{20,15,10,5\}$ and $\beta=0.75$. As above, $C=1.8750 \times 10^{-7}, Y=1.5, G=10, M=20, \tau_{o}=1 /(1,200,000)$ and the parameters for option pricing are $r=5 \%, D=0$ and $S(0)=100$. It is clear that the difference in prices is negligible. 


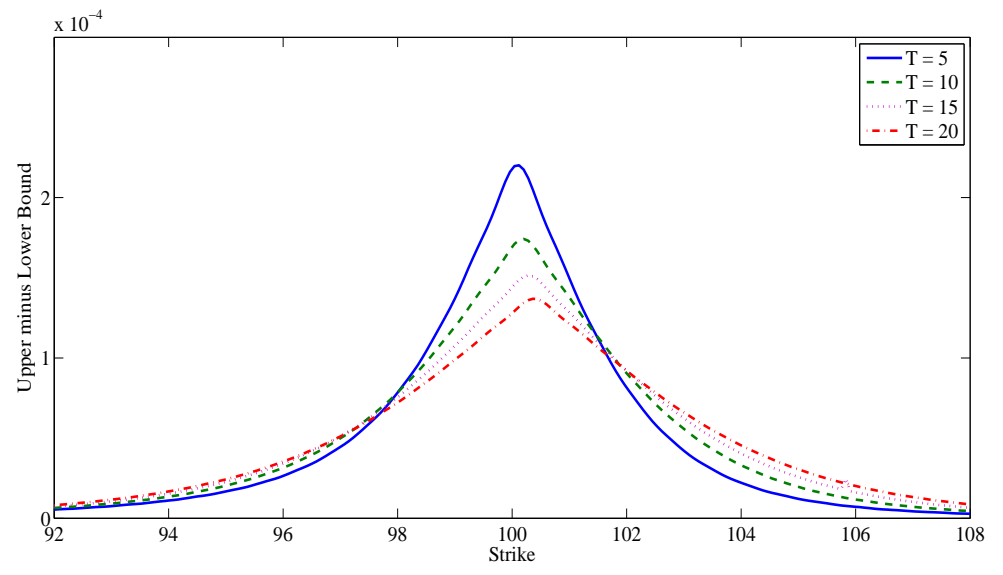

Figure 5. Difference between upper and lower bound from Proposition 4.4 across strikes in the conditionally CGMY model for different days to maturity $T=\{20,15,10,5\}$ and $\beta=0.75$. We use $C=1.8750 \times 10^{-7}, Y=1.5, G=10, M=20$, $\tau_{o}=1 /(1,200,000)$ and the parameters for option pricing are $r=5 \%, D=0$ and $S(0)=100$.

This price difference, and others that were calculated but in the interest of space are not reported here, indicates that the difference between the two prices is indistinguishable in the light of the bid/ask spread and tick sizes observed in financial markets.

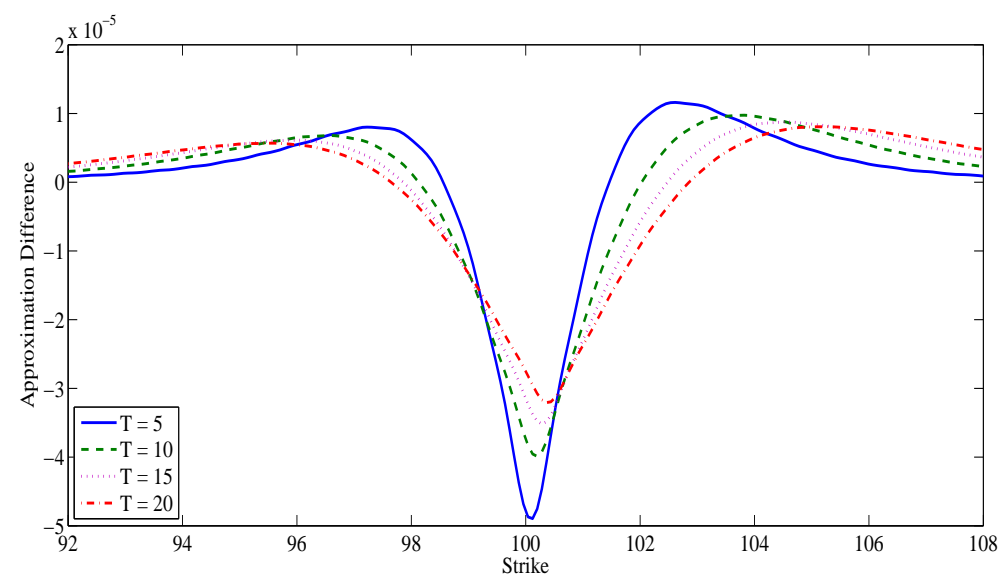

Figure 6. Call option price using $(5.1)$ minus call option price using $(5.2)$ across strikes for conditionally CGMY model for different days to maturity $T=\{20,15,10,5\}$ and $\beta=0.75$. Here $C=1.8750 \times 10^{-7}, Y=1.5, G=10, M=20, \tau_{o}=$ $1 /(1,200,000)$ and the parameters for option pricing are $r=5 \%, D=0$ and $S(0)=100$. 


\section{Conclusions}

Based on tick-by-tick data I propose a non-explosive MPP to model the risk-neutral dynamics of stock prices where the survival function of the trades is the ML function and price revisions possess an infinitely divisible distribution. The ML is a suitable survival function because its right tail exhibits algebraic decay; something that we also observe in the empirical tick-by-tick data and that has been shown in previous studies.

I show that the value of European-style options satisfies a PIDE in time-to-maturity where the time-to-maturity derivative is a non-local operator, instead of the traditional $\partial / \partial T$ local derivative, known as the Caputo operator. This PIDE is new in the options pricing literature and the presence of the Caputo fractional derivative is a consequence of the non-Markovianity present in the arrival of trades in the model. The PIDE I derive can be solved either by using standard numerical methods, see Podlubny (1999), or for vanilla options by employing numerical Fourier inversions which is a widely used technique to price vanilla options when prices are modeled as exponential Lévy processes.

From a financial viewpoint, the model proposed here is interesting because it directly uses tickby-tick data to extract information of trade arrival in high frequency. Although the idea of viewing markets on 'business time' dates back to the work of Clark (1973), explicitly incorporating empirical waiting-times between trades in price dynamics in the form of an MPP is relatively new and explored in the context of option pricing for the first time in Cartea and Meyer-Brandis (2010) and in this paper.

I also show that the infinitesimal operator appearing in the pricing PIDEs coincides with the infinitesimal operator of a Lévy process. At first this is expected because, after all, I assume that the price shocks possess an infinitely divisible distribution. But if we bear in mind that the family of waitingtime distributions used here can only have a finite number of trades within a finite time interval, it is interesting to see that we obtain pricing equations, by using an infinite order approximation of the characteristic function of the MPP, with an infinitesimal generator of a Lévy process that includes infinite activity processes. However I note that the relationship between the non-explosive MPP and Lévy processes, of finite or infinite activity, arises from the distributional properties of the MPP and not the path properties. 


\section{References}

Carr, Peter, Hélyette Geman, Dilip Madan, and Marc Yor, 2002, The Fine Structure of Asset Returns: an Empirical Investigation, Journal of Business 75, 305-332.

Carr, Peter, Hélyette Geman, Dilip Madan, and Marc Yor, 2003, Stochastic Volatility for Lévy Processes, Mathematical Finance 13, 345-382.

Carr, Peter, and Liuren Wu, 2004, Time-Changed Lévy Processes and Option Pricing, Journal of Financial Economics 71, 113-141.

Cartea, Álvaro, and Diego del Castillo-Negrete, 2007, Fractional Diffusion Models of Option Prices in Markets with Jumps, Physica A 374, 749763.

Cartea, Álvaro, and Sam Howison, 2009, Option pricing with Lévy-Stable Processes Generated by Lévy-Stable Integrated Variance, Quantitative Finance 9, 397-409.

Cartea, Álvaro, and Thilo Meyer-Brandis, 2010, How Duration Between Trades of Underlying Securities Affects Option Prices, Review of Finance (in press).

Clark, Peter K., 1973, A Subordinated Stochastic Process Model with Finite Variance for Speculative Prices, Econometrica 41, 135-155.

Cont, Rama, and Peter Tankov, 2004, Financial Modelling With Jump Processes. (Chapman and Hall London) 1 st edn.

Del-Castillo-Negrete, D., B. Carreras, and V. Lynch, 2005, Nondiffusive Transport in Plasma Turbulence: A Fractional Diffusion Approach, Physical Review Letters 94, 065003.

Dufour, Alfonso, and Robert F. Engle, 2000, Time and the Price Impact of a Trade, The Journal of Finance LV, 2467-2498.

Engle, Robert F., 2000, The Econometrics of Ultra-High-Frequency Data, Econometrica 68, 1-22.

Heston, Steve L., 1993, A Closed-Form Solution for Options with Stochastic Volatility with Applications to Bond and Currency Options, The Review of Financial Studies 6, 327-343.

Jacobsen, Martin, 2006, Point Process Theory and Applications. (Birkhäuser).

Kallsen, Jan, and Albert N. Shiryaev, 2002, Time Change Representation of Stochastic Integrals, Theory of Probability and Its Applications 46, 522-528. 
Mainardi, Francesco, Marco Raberto, Rudolf Gorenflo, and Enrico Scalas, 2000, Fractional Calculus and Continuous-time Finance II: the waiting-time distribution, Physica A 287, 469-481.

Podlubny, Igor, 1999, Fractional Differential Equations vol. 198 of Mathematics in Science and Engineering. (Academic Press San Diego, California) 1st edn.

Scalas, Enrico, Rudolf Gorenflo, and Francesco Mainardi, 2000, Fractional Calculus and Continuoustime Finance, Physica A 284, 376-384. 